\title{
Direct costs of severe cutaneous adverse reactions in a tertiary hospital in Korea
}

\author{
Min-Suk Yang ${ }^{1,2}$, Ju-Young Kim²,3, Min-Gyu Kang ${ }^{4}$, Suh-Young Lee ${ }^{5}$, Jae-Woo Jung ${ }^{6}$, Sang-Heon Cho ${ }^{2,3}$, \\ Kyung-Up Min ${ }^{2}$, and Hye-Ryun Kang ${ }^{2,3}$
}

\begin{abstract}
${ }^{1}$ Department of Internal Medicine, Seoul Metropolitan Government Seoul National University Boramae Medical Center, Seoul; ${ }^{2}$ Department of Internal Medicine, Seoul National University College of Medicine, Seoul; ${ }^{3}$ Drug Safety Monitoring Center, Seoul National University Hospital, Seoul; ${ }^{4}$ Department of Internal Medicine, Chungbuk National University College of Medicine, Cheongju; ${ }^{5}$ Department of Internal Medicine, Hallym University Kangdong Sacred Heart Hospital, Seoul; ${ }^{6}$ Department of Internal Medicine, Chung-Ang University Hospital, Seoul, Korea
\end{abstract}

Received: November 10, 2015

Revised : October 25, 2017

Accepted: November 10, 2017

\section{Correspondence to}

Hye-Ryun Kang, M.D.

Department of Internal Medicine, Seoul National University College of Medicine, 101 Daehak-ro, Jongno-gu, Seoul 03080, Korea

Tel: $+82-2-2072-0820$

Fax: +82-2-742-3291

E-mail:helenmed@snu.ac.kr
Background/Aims: There are only a few reports on the direct costs of severe cutaneous adverse reactions (SCARs), including drug reaction with eosinophilia and systemic symptoms (DRESS), Stevens-Johnson syndrome (SJS), and toxic epidermal necrolysis (TEN), despite the tremendous negative impact these reactions can have on patients. We estimated the direct costs of treating SCARs.

Methods: Patients admitted to a tertiary teaching hospital for the treatment of SCARs from January 1, 2005 to December 31, 2010 were included. Patients who had experienced SCARs during their admission for other medical conditions were excluded. The direct costs of hospitalization and outpatient department visits were collected. Inpatient and outpatient care costs were calculated, and factors affecting inpatient care costs were analyzed.

Results: The total healthcare cost for the management of 73 SCAR patients ( 36 with DRESS, 21 with SJS, and 16 with TEN) was 752,067 US dollars (USD). Most of the costs were spent on inpatient care (703,832 USD). The median inpatient care cost per person was 3,720 (range, 1,133 to 107,490) USD for DRESS, 4,457 (range, 1,224 to 21,428) USD for SJS, and 8,061 (range, 1,127 to 52,220) USD for TEN. Longer hospitalization significantly increased the inpatient care costs of the patients with DRESS (by 428 USD [range, 395 to 461] per day). Longer hospitalization and death significantly increased the inpatient care costs of the patients with SJS/TEN (179 USD [range, 148 to 210] per day and an additional 14,425 USD [range, 9,513 to 19,337] for the deceased).

Conclusions: The management of SCARs required considerable direct medical costs. SCARs are not only a health problem but also a significant financial burden for the affected individuals.

Keywords: Stevens-Johnson syndrome; Drug hypersensitivity syndrome; Health care costs; Korea

Min-Suk Yang, Sang-Heon Cho, Kyung-Up Min, and Hye-Ryun Kang: added affiliation 'Institute of Allergy and Clinical Immunology, Seoul National University Medical Research Center, Seoul, Korea'

Ju-Young Kim: current affiliation ‘Department of Internal Medicine, Gyeongsang National University Changwon Hospital, Changwon, Korea'

Suh-Young Lee: current affiliation 'Department of Internal Medicine, Seoul National University College of Medicine and Drug Safety Monitoring Center, Seoul National University Hospital, Seoul, Korea' 


\section{INTRODUCTION}

Adverse drug reactions (ADRs) refer to noxious and unintended drug effects that occur at doses used in humans for prophylaxis, diagnosis, or therapy [1]. ADRs are one of the leading causes of death in United States hospitals [2] and can necessitate hospital admission or prolong the length of hospital stay, which can eventually lead to increased health care costs. In a meta-analysis of 39 prospective studies from the United States over a period of 32 years (1966 to 1996), the overall incidence of ADRs in hospitalized patients was $15.1 \%$, and that of serious ADRs was 6.7\% [2]. The incidence was calculated as the sum of both ADRs that resulted in hospitalization and those that occurred during hospitalization for other diseases. Several reports on the costs associated with ADRs have shown that they are substantially high [3-5]. However, the estimation of costs can be difficult because of the heterogeneity of ADRs, and indeed, the costs were found to be quite variable and dependent on the type of ADR and culprit agent involved [6].

Severe cutaneous adverse reactions (SCARs) such as Stevens-Johnson syndrome (SJS), toxic epidermal necrolysis (TEN), and drug reaction with eosinophilia and systemic symptoms (DRESS) are rare but potentially fatal diseases. Due to their unexpected drastic progression and serious outcomes, SCARs can be a considerable financial burden to the affected patients and their families. Moreover, standard treatments other than supportive care are not well established, and costly experimental treatments such as intravenous immunoglobulin (IVIG) have often been used, adding to the costs. However, despite its potentially negative impact, the financial burden of SCARs on patients has been overlooked because of the rarity of these diseases [5-7]. In this study, the authors aimed to determine the direct costs of SCARs in a tertiary hospital in Korea.

\section{METHODS}

Seventy-three patients with SCARs (36 DRESS, 21 SJS, and 16 TEN cases) admitted to the Seoul National University Hospital from January 1, 2005 to December 31, 2010 were selected by International Classification of Diseases 10 (ICD-10) diagnostic code and retrospective review of medical records [8]. The diagnostic criteria proposed by the RegiSCAR study group were used to define the individual cases $[9,10]$. In short, SJS and TEN were diagnosed when there were typical skin lesions such as widespread erythematous or purpuric macules or flat atypical targets. SJS and TEN were diagnosed when the area of skin detachment was less than $10 \%$ and greater than $30 \%$ of the total body surface area, respectively. There was no case of SJS-TEN overlap among our subjects [9]. DRESS was diagnosed according to the presence of typical skin lesions, fever, enlarged lymph nodes, hematologic abnormality, and organ involvement [10]. The causative agents were assessed based on the WHO-UMC (World Health Organization-Uppsala Monitoring Centre) causality assessment system. Patients who had been admitted for other medical conditions before the onset of SCARs were excluded. Clinical characteristics regarded as relevant to treatment costs, such as duration of hospitalization, admission to an intensive care unit (ICU), use of IVIG, and the presence of ocular involvement, were collected.

Data on the direct costs of SCARs were extracted from a hospital accounting system and divided into two categories: inpatient care costs and outpatient care costs. Inpatient care costs included expenses during a hospital stay such as costs for room and board, physician services, laboratory tests, imaging tests, procedures, and medications. Inpatient care costs were further divided into reimbursed and out-of-pocket costs.

Outpatient care costs consisted of the total payment for problems related to SCARs. Emergency department visits that did not result in readmission were also considered as outpatient visits. The terms related to direct costs used in this article are summarized in Table 1.

\section{Statistical analysis}

Statistical analyses were performed using IBM SPSS Statistics version 20 for Windows (IBM Co., Armonk, NY, USA). Normally distributed variables were expressed as mean \pm standard deviation and non-normally distributed variables as medians and ranges. Costs for outpatient visits were estimated in person-year units. Subdivision cost percentages were estimated by dividing the aggregate cost of each subdivision by the total medical cost of the entire population. All costs were expressed in US dollars (USD). One USD was equivalent 
Table 1. Terms used in this article

\begin{tabular}{ll}
\hline Terms used & \multicolumn{1}{c}{ Description } \\
\hline Total healthcare cost & The total cost of treating patients with each disease \\
Cost per person & The cost of treating one patient with a specific disease \\
Inpatient care cost & The cost of treating SCARs during hospitalization \\
Outpatient care cost & The cost of treating SCARs and their sequelae in outpatient departments \\
Reimbursed cost & The amount that the National Health Insurance pays to the hospital \\
Out-of-pocket cost & The amount that the patient pays to the hospital directly \\
\hline
\end{tabular}

SCAR, severe cutaneous adverse reaction.

Table 2. Demographic characteristics of the study population

\begin{tabular}{|c|c|c|c|}
\hline Characteristic & $\operatorname{DRESS}(\mathrm{n}=36)$ & SJS $(n=21)$ & $\operatorname{TEN}(\mathrm{n}=16)$ \\
\hline Age, yr & $48.1 \pm 21.7$ & $34.3 \pm 21.9$ & $42.8 \pm 27$ \\
\hline Male sex & $15(41.7)$ & $9(42.9)$ & $9(56.2)$ \\
\hline \multicolumn{4}{|l|}{ Outcome } \\
\hline Recovery & $35(97.2)$ & $21(100)$ & $12(75)$ \\
\hline Death & $1(2.8)$ & & $4(25)$ \\
\hline Admission to ER & $6(16.7)$ & $2(9 \cdot 5)$ & $2(12.5)$ \\
\hline Admission to ICU & $2(5.6)$ & $3(14 \cdot 3)$ & $4(25)$ \\
\hline Duration of hospitalization, day & $14(3-218)$ & $20(8-60)$ & $21.5(10-292)$ \\
\hline Use of IVIG & NA & $9(42.9)$ & $10(62.5)$ \\
\hline Total amount of IVIG, g & NA & $30(10-124)$ & $35(13-138)$ \\
\hline Use of steroids & $33(91.7)$ & $17(81)$ & $10(62.5)$ \\
\hline Highest doses of systemic steroids, mg/day ${ }^{\mathrm{a}}$ & $44(12-188)$ & $32(7-125)$ & $55(24-375)$ \\
\hline Duration of steroid treatment, day & $51.5(4-1,295)$ & $21(1-152)$ & $15(1-118)$ \\
\hline \multicolumn{4}{|l|}{ Causative agents } \\
\hline Allopurinol & $12(33 \cdot 3)$ & $3(14 \cdot 3)$ & $2(12.5)$ \\
\hline Anti-epileptic drugs & $9(25)$ & $4(19)$ & $2(12.5)$ \\
\hline Antibiotics & $6(16.7)$ & $1(4.8)$ & $2(12.5)$ \\
\hline Non-antibiotic sulfonamides ${ }^{b}$ & $2(5.6)$ & $2(12.5)$ & $3(25)$ \\
\hline Others & $7(19.4)$ & $11(47.6)$ & $7(43.8)$ \\
\hline
\end{tabular}

Values are presented as mean $\pm \mathrm{SD}$, number (\%), or median (range).

DRESS, drug reaction with eosinophilia and systemic symptoms; SJS, Stevens-Johnson syndrome; TEN, toxic epidermal necrolysis; ER, emergency room; ICU, intensive care unit; IVIG, intravenous immunoglobulin; NA, not applicable.

${ }^{a}$ Values are expressed in equivalent doses of methylprednisolone.

${ }^{\mathrm{b}}$ Non-antibiotic sulfonamides included sulfasalazine, mesalazine, methazolamide, and furosemide.

to approximately 1,050 Korean won at the time of this study. Adjustment for inflation was not considered. The Kruskal-Wallis test was used to compare the costs for managing SJS, TEN, and DRESS. A p value less than 0.05 was considered statistically significant. Factors affecting inpatient care costs were analyzed for DRESS and SJS/
TEN, separately. To identify the factors affecting inpatient care costs for DRESS, linear regression analysis was performed, adjusting for sex, age, length of hospitalization, and presence of renal and hepatic involvement. To identify the factors affecting inpatient care costs for SJS/ TEN, linear regression analysis was performed, adjust- 
ing for sex, age, disease severity, length of hospitalization, IVIG usage, and death. This study was approved by the Seoul National University Hospital Institutional Review Board (IRB No. 1302-080-467). IRB approved the waiver to document informed consent for this study.

\section{RESULTS}

\section{Demographic and clinical characteristics of the study subjects}

The demographic characteristics of 73 subjects with SCARs included in this study are shown in Table 2. The leading causative agents were allopurinol $(n=17,23.3 \%)$, anti-epileptic drugs (AEDs, $\mathrm{n}=15,20.5 \%$ ), and antibiotics ( $\mathrm{n}=9,12.3 \%$ ). The median length of hospital stay was 2 weeks for DRESS and approximately 3 weeks for SJS and TEN. However, the length of hospital stay varied widely from 3 to 292 days. There were five fatalities, including four patients with TEN and one patient with DRESS. Twelve patients (two with DRESS [2/36, 5.6\%], three with SJS [3/21, 14.3\%], and four with TEN [4/16, 25\%]) were admitted to the ICU, and one of the TEN cases died. More than $90 \%$ of DRESS patients received systemic corticosteroids, and $61 \%$ (22 of 36 ) received them for more than 7 weeks. Patients with SJS/TEN had lower doses and a shorter duration of systemic steroid treatment than did cases with DRESS. IVIG was used only for SJS/TEN cases and was administered to $42.9 \%$ with SJS and $62.5 \%$ with TEN.

\section{Total healthcare costs for SJS/TEN and DRESS}

The total healthcare cost for managing 73 SCAR patients was 752,067 USD. The three most common causative drugs (allopurinol, AEDs, and antibiotics) accounted for $49 \%$ of the total cost. After categorizing by disease entity, 167,957 USD was spent on 21 SJS patients, 286,745 USD on 16 TEN patients, and 297,365 USD on 36 patients with DRESS. Most of the costs were spent on inpatient care (703,832 USD): 153,699 USD for SJS, 364,700 USD for TEN, and 285,434 USD for DRESS. Out-of-pocket costs accounted for approximately $45 \%$ of the inpatient care costs: 84,898 USD (55\%) for SJS, 114,317 USD (43\%) for TEN, and 116,039 USD (41\%) for DRESS. Costs for room and board accounted for the majority $(225,885$ USD, $32 \%$ ) of the total healthcare cost, followed by costs for medications $(178,413 \mathrm{USD}, 25 \%)$ and laboratory tests (142,077 USD, 20\%). Costs for procedures, imaging tests, and physician services followed in that order. A considerable portion of the cost for medications for SJS and TEN was not covered by insurance. Notably, the cost for IVIG $(36,163$ USD) accounted for a large portion of the total cost $(111,411$ USD) for medications in SJS and TEN patients. Sixty-two patients (85\%) visited the outpatient department after discharge, and the total outpatient care cost was estimated to be 48,234 USD: 14,259 USD for SJS, 22,045 USD for TEN, and 11,931 USD for DRESS. Costs for outpatient care with the patients' main physicians, such as internists, dermatologists, and pediatricians, were 13,063 USD, and costs for visits to other departments, such as ophthalmology, the pain clinic, urology, neuropsychiatry, and other internal medicine departments, were 30,345 USD. In particular, SJS and TEN patients spent approximately half $(24,422$ USD) of their outpatient care costs on ophthalmological care.

\section{Inpatient care cost per person}

The median inpatient cost per person was 3,720 USD (range, 1,133 to 107,490) for DRESS, 4,457 USD (range, 1,224 to 21,428 ) for SJS, and 8,061 USD (range, 1,127 to $52,220)$ for TEN. The median out-of-pocket cost per person was 1,660 USD (range, 460 to 28,125) for DRESS, 3,559 USD (range, 627 to 13,811) for SJS, and 5,049 USD (range, 485 to 18,616$)$ for TEN. There was no significant difference in costs per person among SJS, TEN, and DRESS patients (Table 3). Linear regression analysis was performed to assess the factors affecting the inpatient care cost. According to the univariate analysis, the only factor that affected the inpatient care cost for DRESS was the length of hospitalization $(p<0.001)$. The presence of renal involvement showed a marginal relationship $(p$ $=0.07$ ). However, only the length of hospitalization significantly increased the inpatient care cost (by 428 USD [range, 395 to 461 ] per day) in the multivariate analysis. The severity of disease, the length of hospitalization, the usage of IVIG, and death were significantly related to the inpatient care cost for SJS/TEN in the univariate analysis. According to the multivariate analysis, the length of hospitalization and death were significantly associated with increased inpatient care cost. The inpatient care cost increased by 179 USD (range, 148 to 210) per day, and the survivors paid 14,425 USD (range, 9,513 to 19,337) 
Table 3. Direct costs per person for severe cutaneous adverse reactions

\begin{tabular}{lcccc}
\hline & DRESS $(\mathrm{n}=36)$ & SJS $(\mathrm{n}=21)$ & TEN $(\mathrm{n}=16)$ & $p$ value \\
\hline Inpatient care cost & $3,720(1,133-107,490)$ & $4,457(1,224-21,428)$ & $8,061(1,127-52,220)$ & 0.07 \\
Reimbursed cost & $1,887(691-84,134)$ & $2,364(0-10,002)$ & $3,275(653-39,647)$ & 0.06 \\
Out-of-pocket cost & $1,660(460-28,125)$ & $3,559(627-13,811)$ & $5,049(485-18,616)$ & 0.10 \\
$\begin{array}{l}\text { Outpatient care cost } \\
\text { (person-year) }\end{array}$ & $291(0-7,197)$ & $482(0-1,799)$ & $383(0-2,206)$ & 0.48 \\
\hline
\end{tabular}

Values are presented as medians (range). Costs are expressed in US dollars (USD).

DRESS, drug reaction with eosinophilia and systemic symptoms; SJS, Stevens-Johnson syndrome; TEN, toxic epidermal necrolysis.

Table 4. Factors that affected the inpatient care cost of managing severe cutaneous adverse reactions

\begin{tabular}{|c|c|c|c|c|c|c|}
\hline & \multicolumn{3}{|c|}{ Univariate } & \multicolumn{3}{|c|}{ Multivariate } \\
\hline & Coefficient $^{\mathrm{a}}$ & SE & $p$ value & $\beta$ Coefficient $^{\mathrm{a}}$ & $\mathrm{SE}$ & $p$ value \\
\hline \multicolumn{7}{|l|}{$\operatorname{DRESS}(\mathrm{n}=16)$} \\
\hline Sex & 7,684 & 5,846 & 0.20 & 345 & 2,342 & 0.88 \\
\hline Age & -206 & 133 & 0.13 & -63 & 54 & 0.25 \\
\hline Length of hospitalization & 443 & 29 & $<0.01$ & 428 & 33 & $<0.01$ \\
\hline Renal involvement & 13,373 & 7,104 & 0.07 & 2,363 & 3,040 & 0.44 \\
\hline Hepatic involvement & 6,500 & 6,049 & 0.29 & -225 & 2,413 & 0.92 \\
\hline \multicolumn{7}{|l|}{ SJS/TEN $(\mathrm{n}=37)$} \\
\hline Sex & 3,650 & 4,256 & 0.86 & 2,677 & 2,819 & 0.35 \\
\hline Age & -121 & 88 & 0.18 & -24 & 61 & 0.70 \\
\hline Severity of disease & 8,805 & 4,076 & 0.04 & 707 & 3,109 & 0.82 \\
\hline Length of hospitalization & 186 & 35 & $<0.01$ & 179 & 31 & $<0.01$ \\
\hline IVIG & 10,441 & 3,922 & 0.01 & 4,694 & 2,906 & 0.12 \\
\hline Death & 17,032 & 6,296 & 0.01 & 14,425 & 4,912 & 0.01 \\
\hline
\end{tabular}

SE, standard error; DRESS, drug reaction with eosinophilia and systemic symptoms; SJS, Stevens-Johnson syndrome; TEN, toxic epidermal necrolysis; IVIG, intravenous immunoglobulin.

${ }^{a}$ From linear regression analysis.

less than the deceased (Table 4).

\section{Outpatient care cost per person}

Although several patients paid more than 1,000 USD for follow-up visits, outpatient care costs were generally much less than inpatient care costs. However, the duration of outpatient care differed significantly (663.5 days [range, 26 to 2,572] for patients with ocular involvement vs. 34 days [range, o to 1,600] for those without, $p<$ 0.001 ) in SJS and TEN patients. In consequence, patients with ocular involvement paid more money for outpatient care than did those without (712 USD [range, 186 to 15,378] for patients with ocular involvement vs. 21 USD [range, o to 933] for those without, $p<0.001$ ).

\section{DISCUSSION}

This is the first study on the direct medical costs associated with treating SCARs in Korea. In the present study, the median inpatient care cost per person was 3,720 USD for DRESS, 4,457 USD for SJS, and 8,061 USD for TEN. Most of the costs were spent on inpatient care. The length of hospitalization increased the inpatient care cost by 428 USD for DRESS. The inpatient care cost for SJS/TEN increased by 179 USD per day, and the survi- 
vors paid less than the deceased.

The findings from this study show that the cost of managing SCARs was quite high. There have been only a few reports on this specific topic. Schlienger et al. [11] determined the direct cost of inpatient care in $13 \mathrm{Ca}-$ nadian patients with severe adverse reactions to AEDs treated at one institution to be a median 3,128 Canadian dollars $(2,283$ USD). They estimated that the average annual direct cost for severe ADRs due to AEDs was 11,003 Canadian dollars ( 8,032 USD). In India, Barvaliya and et al. [7] determined the direct costs of managing SJS and TEN for 11 SJS, 15 TEN, and 6 SJS-TEN overlap patients to be 2,460 Rupees ( 39 USD), 7,910 Rupees ( 27 USD), and 4,857 Rupees ( $\sim 77$ USD), respectively. The direct costs of managing SCARs in India were lower than expected. This may be because the authors estimated the costs for drugs, investigations, and consumables during the entire hospital stay, but costs for nursing and room, which could account for a substantial proportion of total medical costs, were not included. The differences between our results and those of the other reports may be explained by the different patient populations and different items included in the medical cost estimation. They may also be explained by the different economic conditions and insurance systems of each country. For example, the total medical expenditure per person in Korea is approximately 1,700 USD, whereas that in Canada is 4,000 USD [12]. The direct cost of managing SCARs in Canada per person is approximately 8,000 USD, which is double the total medical expenditure per person. In Korea, the direct cost of managing SCARs was approximately 4,400 USD, which was more than double the total medical expenditure per person. Thus, the cost of managing SCARs in Korea can be considered relatively more burdensome than that in Canada.

A considerable portion of the inpatient care costs for managing SJS and TEN was not covered by insurance. Patients with SJS, TEN, and DRESS paid approximately $55 \%, 43 \%$, and $41 \%$ of their inpatient care costs, respectively. In particular, the cost for IVIG, which accounted for approximately one-third of the cost of medication for SJS/TEN patients, caused a considerable burden to the patients. The length of hospitalization was the single most important factor that affected the inpatient care cost for both DRESS and SJS/TEN. Fatality greatly increased the inpatient care costs for SJS/TEN. Other fac- tors such as ICU admission and ocular involvement did not affect inpatient care cost.

According to Statistics Korea, chronic renal disease is the most expensive disease to treat, requiring an annual expense per capita of more than 8,500 USD, followed by lung and liver/biliary cancers ( $\sim, 700$ USD), intracranial hemorrhage $(\sim 4,800 \mathrm{USD})$, and breast cancer $(\sim 3,800$ USD). The inpatient care costs for TEN, SJS, and DRESS from our study were 8,061 USD, 4,457 USD, and 3,720 USD, respectively. Although it is not possible to compare these costs directly, it can be inferred that costs for treating one SCAR case were comparable with those for treating the national top five most expensive common medical conditions.

This study has some limitations. First, due to the retrospective design, we could only estimate the direct costs of SCARs and could not assess the indirect or intangible costs. Second, because this study was conducted in one tertiary hospital, the results do not accurately represent situations in other hospitals; the patient-care patterns may be very dependent on the physicians. To compensate for this, we analyzed national data for all Korean SJS/TEN patients. Finally, the results cannot be generalized to other countries because of differences in medical service systems. Nonetheless, this study may increase awareness of the seriousness of the financial aspect associated with management of SCARs.

In conclusion, SCARs are not only serious health problems but also significant financial burdens accounting for considerable direct medical costs.

\section{KEY MESSAGE}

1. The management of severe cutaneous adverse reactions (SCARs) required considerable direct medical costs per person, which were comparable with the costs for other major health problems.

2. The cost of managing toxic epidermal necrolysis (TEN) was the highest among the SCARs evaluated, followed by Stevens-Johnson syndrome (SJS) and drug reaction with eosinophilia and systemic symptoms (DRESS).

3. SJS and TEN patients with ocular involvement spent a considerable amount of money even after their recovery. 


\section{Conflict of interest}

No potential conflict of interest relevant to this article was reported.

\section{Acknowledgments}

This research was supported by a grant from Ministry of Food and Drug Safety to operation of the regional pharmacovigilance center in 2014 .

\section{REFERENCES}

1. World Health Organization. International Drug Monitoring: the Role of National Centers. Geneva: WHO, 1972.

2. Lazarou J, Pomeranz BH, Corey PN. Incidence of adverse drug reactions in hospitalized patients: a meta-analysis of prospective studies. JAMA 1998;279:1200-1205.

3. Bates DW, Spell N, Cullen DJ, et al. The costs of adverse drug events in hospitalized patients. Adverse Drug Events Prevention Study Group. JAMA 1997;277:307-311.

4. Izquierdo E, Rodriguez C, Pampliega E, Filinger E. Determination of the frequency and direct cost of the adverse drug events in Argentina. Curr Drug Saf 2009;4:103-106.

5. Bordet R, Gautier S, Le Louet H, Dupuis B, Caron J. Analysis of the direct cost of adverse drug reactions in hospitalised patients. Eur J Clin Pharmacol 2001;56:935-941.

6. Gautier S, Bachelet H, Bordet R, Caron J. The cost of adverse drug reactions. Expert Opin Pharmacother 2003;4:319-326.

7. Barvaliya M, Sanmukhani J, Patel T, Paliwal N, Shah H, Tripathi C. Drug-induced Stevens-Johnson syndrome (SJS), toxic epidermal necrolysis (TEN), and SJS-TEN overlap: a multicentric retrospective study. J Postgrad Med 2011;57:115-119.

8. Yang MS, Kang MG, Jung JW, et al. Clinical features and prognostic factors in severe cutaneous drug reactions. Int Arch Allergy Immunol 2013;162:346-354.

9. Bastuji-Garin S, Rzany B, Stern RS, Shear NH, Naldi L, Roujeau JC. Clinical classification of cases of toxic epidermal necrolysis, Stevens-Johnson syndrome, and erythema multiforme. Arch Dermatol 1993;129:92-96.

10. Kardaun SH, Sidoroff A, Valeyrie-Allanore L, et al. Variability in the clinical pattern of cutaneous side-effects of drugs with systemic symptoms: does a DRESS syndrome really exist? Br J Dermatol 2007;156:609-611.

11. Schlienger RG, Oh PI, Knowles SR, Shear NH. Quantifying the costs of serious adverse drug reactions to antiepileptic drugs. Epilepsia 1998;39 Suppl 7:S27-S32.

12. OECD iLibrary. Expenditure on health [Internet]. Paris: OECD, c2017 [cited 2018 Jan 5]. Available from: http:// www.oecd-ilibrary.org/social-issues-migration-health/ health-key-tables-from-oecd_20758480;jsessionid=1h286tlwsidhy.x-oecd-live-o1. 